\title{
Retinal Waves Trigger Spindle Bursts in the Neonatal Rat Visual Cortex
}

\author{
Ileana L. Hanganu, Yehezkel Ben-Ari, and Rustem Khazipov \\ Institut de Neurobiologie de la Mediteranée, Université de la Mediteranée, Institut National de la Santé et de la Recherche Médicale U29, 13273 Marseille, \\ France
}

During visual system development, the light-insensitive retina spontaneously generates waves of activity, which are transmitted to the lateral geniculate nucleus. The crucial question is whether retinal waves are further transmitted to the cortex and influence the early cortical patterns of activity. Using simultaneous recordings from the rat retina and visual cortex during the first postnatal week in vivo, we found that spontaneous retinal bursts are correlated with spindle bursts (intermittent network bursts associated with spindle-shape field oscillations) in the contralateral visual cortex (V1). V1 spindle bursts could be evoked by electrical stimulation of the optic nerve. Intraocular injection of forskolin, which augments retinal waves, increased the occurrence of $\mathrm{V} 1$ spindle bursts. Blocking propagation of retinal activity, or removal of the retina reduced the frequency, but did not completely eliminate the cortical spindle bursts. These results indicate that spontaneous retinal waves are transmitted to the visual cortex and trigger endogenous spindle bursts. We propose that the interaction between retinal waves and spindle bursts contributes to the development of visual pathways to the cortex.

Key words: development; in vivo; delta brush; oscillations; forskolin; patch clamp

\section{Introduction}

Spontaneous bursts of correlated neuronal activity in immature neuronal networks is a hallmark of the developing nervous system (Ben-Ari, 2001). Growing evidence indicates that early patterns of coordinated neuronal activity are implicated in a number of developmental processes including synaptogenesis, neuronal differentiation and migration, axonal refinement, and formation of early networks (Katz and Shatz, 1996; Moody and Bosma, 2005). In the visual system, early stages of development are characterized by the presence of spontaneous waves of activity in the retina before it can respond to light. Retinal waves are generated in the network of retinal ganglion and amacrine cells and synchronize most of retinal activity (Galli and Maffei, 1988; Meister et al., 1991; Wong et al., 1993; Torborg and Feller, 2005). Using an original in vitro preparation of the neonatal mouse intact retina and lateral geniculate nucleus (LGN), it was demonstrated that spontaneous retinal activity is transmitted via the optic nerve to the LGN, where it drives bursts of activity (Mooney et al., 1996). Modulation of retinal waves during the first postnatal week results in alteration of retinal projections to their subcortical targets, suggesting an instructive role for retinal waves in the devel-

\footnotetext{
Received Feb. 20, 2006; revised April 20, 2006; accepted May 17, 2006.

This work was supported by grants from the Deutsche Forschungsgemeinschaft (I.L.H.), Institut National de la Santé et de la Recherche Médicale, La Fondation pour la Recherche Médicale, and ANR (R.K., Y.B.-A.). We thank W. Kilb, E. Dupont, G. Buzsaki, A. Sirota, and M. Colonnese for helpful comments on this manuscript and N. Ferrand for technical assistance.

Correspondence should be addressed to Rustem Khazipov, Institut de Neurobiologie de la Mediteranée, Université de la Mediteranée, Institut National de la Santé et de la Recherche Médicale U29, Avenue de Luminy, B.P. 13, 13272 Marseille, France. E-mail: khazipov@inmed.univ-mrs.fr.

DOI:10.1523/JNEUROSCI.0752-06.2006

Copyright $\odot 2006$ Society for Neuroscience $\quad 0270-6474 / 06 / 266728-09 \$ 15.00 / 0$
}

opment of retinogeniculate connectivity (Shatz and Stryker, 1988; Penn et al., 1998; Muir-Robinson et al., 2002; Stellwagen and Shatz, 2002; Grubb et al., 2003; McLaughlin et al., 2003; Chandrasekaran et al., 2005; Mrsic-Flogel et al., 2005). Evidence also exists for the contribution of retinal waves to cortical development. Ocular dominance columns (ODCs) are formed already in utero before visual experience (Rakic, 1976). Although enucleation experiments suggest that retinal input may not be required for the formation of ODCs (Crowley and Katz, 1999), the complete blockade of retinal activity can disturb segregation of thalamocortical connections in ODCs (Stryker and Harris, 1986). In the neonatal mouse, suppression of retinal waves during the first postnatal week results in imprecise geniculocortical mapping, suggesting that spontaneous retinal waves are also involved in the development of thalamic connections to the visual cortex (Cang et al., 2005). These findings raise the hypothesis that retinal waves are transmitted to and trigger activity in the developing visual cortex. Later during development, a tight correlation exists between visual sensory input and cortical activity, as demonstrated by recordings from postnatal day 22 (P22) to P39 ferrets, where correlated bursts of activity in the LGN and visual cortex could be triggered by light stimulation of the retina (Weliky and Katz, 1999; Chiu and Weliky, 2001; Chiu and Weliky, 2002). However, little is known about the patterns of activity expressed in the visual cortex and the influence of spontaneous retinal waves on cortical activity during the first postnatal week, before the retina becomes responsive to light. In the present study, we used simultaneous in vivo recordings from the retina and visual cortex as well as pharmacological manipulations of retinal activity to determine the impact of retinal waves on the activity of visual cortex in rats during the first postnatal week. We 
provide evidence that spontaneous retinal waves are transmitted to the visual cortex and trigger the dominant endogenous oscillatory pattern of spindle bursts.

\section{Materials and Methods}

All experiments were conducted in accordance with the national laws for the use of animals in research and approved by the local ethical committee. Neocortical recordings were performed from the visual cortex (V1; $0-1.5 \mathrm{~mm}$ anterior to lambda suture and $1.5-4 \mathrm{~mm}$ from the midline) of P5 to P6 rats using the experimental setup and conditions described previously (Leinekugel et al., 2002; Khazipov et al., 2004). For certain investigations, pups aged from P1 to P6 were used. Briefly, under urethane $(0.5-1.5 \mathrm{~g} / \mathrm{kg})$ or deep ice-cooling anesthesia, the heads of the pups were fixed into the stereotaxic apparatus using two metal bars fixed with dental cement on the nasal and occipital bones, respectively. The bodies of the pups were surrounded by a cotton nest and kept at a constant temperature of $37^{\circ} \mathrm{C}$ by a temperature controller (TC-344B; Warner Instruments, Hamden, CT). After a 30-60 min recovery period, single (50 $\mu \mathrm{m}$ diameter) or arrays of tungsten electrodes (50 $\mu \mathrm{m}$ diameter, $500-$ $1000 \mu \mathrm{m}$ separation; A-M Systems, Carlsborg, WA) were inserted vertically into the visual cortex to obtain simultaneous recordings of field potential and multiple-unit activity (MUA) at different depths and locations. One or two silver wires were inserted into the cerebellum or somatosensory cortex (S1) and served as ground electrodes. Stimulation of the optic nerve $(\mathrm{ON})$ was performed after surgically opening the lid under local anesthesia. Electrical pulses $(50-100 \mathrm{~V}, 50 \mu \mathrm{s}, 0.1 \mathrm{~Hz})$ were applied via a bipolar tungsten electrode (160 $\mu \mathrm{m}$ diameter; California Fine Wire Company, Grover Beach, CA) inserted 3-4 mm through the cornea to reach the head of ON. Low-frequency stimulation was used to prevent accommodation. Patch-clamp whole-cell recordings were performed according to the procedure described previously (Margrie et al., 2002; Khazipov et al., 2004) in the vicinity of the extracellular electrode using an Axopatch 200B amplifier (Molecular Devices, Union City, CA). Recording electrodes were pulled from borosilicate glass (Harvard Apparatus, Edenbridge, UK) and were filled with an electrode solution containing the following (in mM): 135 Cs-gluconate, $2 \mathrm{MgCl}_{2}, 0.1 \mathrm{CaCl}_{2}, 1$ EGTA, and $10 \mathrm{HEPES}$, pH 7.2 with $\mathrm{CsOH}, 285$ mOsm. All potentials were corrected for liquid junction potential with $-10 \mathrm{mV}$. Series resistances ranged between 10 and $50 \mathrm{M} \Omega$. Morphological identification of the recorded cells was performed by adding $0.5 \%$ biocytin in the patch pipette and staining the re-sliced ( $300 \mu \mathrm{m}$ thick) paraformaldehydefixed brain for biocytin according to the previously described protocol (Khazipov et al., 2001). Biocytin-stained neurons were analyzed in their somatodendritic properties using an Axioskop microscope (Zeiss, Oberkochen, Germany). In some experiments, the exact depth of extracellular recordings was assessed by using DiI (1, $1^{\prime}$-dioctadecyl-3,3,3', $3^{\prime}$ tetramethyl indocarbocyanine; Invitrogen, Eugene, OR) covered electrodes.

Retinal activity was recorded with a single tungsten electrode (50 $\mu \mathrm{m}$ diameter) advanced maximally $3 \mathrm{~mm}$ through the vitreous after surgically opening one lid of ice-cooling-anesthetized pups. Urethane anesthesia was not used in these recordings because it almost completely abolished the retinal activity ( $n=4$ pups) (Mooney et al., 1996). The ocular muscles were cut and the eye was covered with $2 \%$ agar to minimize movement. Placement of piezoelectric movement detectors in direct contact with the eye allowed discarding artifacts attributable to eye movements. Pharmacological alteration of spontaneous retinal activity was performed on pups fixed on the stereotaxic apparatus. For this, a 26 gauge needle (WPI, Sarasota, FL) attached to a microsyringe pump controller (Micro4; WPI) was used to inject intraocularly $1 \mu$ l solution at the rate of $0.5 \mu \mathrm{l} / \mathrm{min}$. The injected substances were purchased as follows: tetrodotoxin citrate (TTX), forskolin ( $7 \beta$-acetoxy-8,13-epoxy- $1 \alpha, 6 \beta, 9 \alpha$ trhydroxylabd-14-en-11-one), dehydro- $\beta$-erythroidine (DH $\beta \mathrm{E}), R(-)$-3(2-carboxypiperazin-4-yl)-propyl-1-phosphonic acid (CPP), and 6-cyano7-nitroquinoxaline-2,3-dione (CNQX) from Sigma-Aldrich (Lyon, France), and 6-(3-dimethyl aminopropionyl) forskolin hydrochloride (NKH477) (water-soluble forskolin) from Tocris (Ellisville, MO). Stock solutions of the drugs were prepared in water (TTX, DH $\beta$ E, CPP, NKH477) or DMSO (CPP and forskolin), stored at $-20^{\circ} \mathrm{C}$, and diluted in saline on the day of experiment. Control recordings were performed before and after lid opening, after needle insertion, and after solvent injection in each investigated pup. After each injection, the needle was withdrawn and a 10-20 min recovery period was allowed. At the end of pharmacological manipulations, the eye region was additionally anesthetized by ice-cooling, the vitreous was extracted, and one or both retinas were removed with a thin cotton-covered bar. In this case, the recovery period was extended to $30 \mathrm{~min}$.

Data were acquired at a sampling rate of $10 \mathrm{kHz}$ and analyzed off-line using ClampFit software (Molecular Devices). Oscillatory events were detected by eye and confirmed after filtering the raw data between 5 and $40 \mathrm{~Hz}$ using a Butterworth filter. Only events lasting $>100 \mathrm{~ms}$ and containing $>3$ cycles were considered for analysis. Morlet color-coded wavelet spectra were produced using Auto Signal 1.52 software (SPSS, Chicago, IL) and minimal and maximal intensities in power were displayed in red and white, respectively. MUA was analyzed after filtering at $200 \mathrm{~Hz}$ high-pass filter. Retinal bursts were detected using Mini Analysis Program (Synaptosoft, Leonia, NJ) and cortical bursts were considered as being correlated with retinal ones if they occurred within a time window of retinal burst $\pm 1 \mathrm{~s}$. Spontaneous postsynaptic currents (PSCs) were detected and analyzed with Mini Analysis Program. Data are presented as mean \pm SEM. Statistical analyses were performed with Systat (SPSS) and Origin 7 software (OriginLab, Northampton, MA) using Student's $t$ test and Wilcoxon test.

\section{Results}

\section{Retinal bursts and spindle bursts in the visual cortex}

To gain a first indication as to the relationship between retinal and cortical activity, we recorded the activity expressed in the retina and visual cortex in vivo. Whereas eye operation and electrode insertion were performed under ice-cooling anesthesia, retinal activity was explored after recovery from anesthesia in awake P5-P6 pups using extracellular recordings of multiple unit activity. In keeping with previous in vivo and in vitro recordings (Galli and Maffei, 1988; Meister et al., 1991; Wong et al., 1993; Torborg and Feller, 2005), retinal activity was synchronized in intermittent bursts of action potentials lasting for $3.5 \pm 0.4 \mathrm{~s}$ ( $n=6$ pups), followed by silent periods with occasional single spiking (Fig. $1 A)$. However, the bursts occurred more frequently $(3.1 \pm 0.7 /$ $\min ; n=6$ pups) than in the retina in vitro (Wong et al., 1995; Mooney et al., 1996; Firth et al., 2005). According to previous multielectrode array and imaging studies from the retina in vitro, each burst represents local excitation of neurons in the vicinity of the recording site by the wave of activity spreading across the retina (Wong et al., 1995; Firth et al., 2005; Torborg and Feller, 2005).

Activity in the visual cortex was explored using extracellular and patch-clamp recordings (P5-P6; $n=32$ pups). Similar to observations in the somatosensory cortex (Khazipov et al., 2004), intermittent spindle bursts were the dominant field pattern of activity in the immature visual cortex (Fig. $1 B$, top traces). These field potential oscillations had a mean average amplitude of $75.7 \pm 5.3 \mu \mathrm{V}$ and a frequency of $11.9 \pm 0.5 \mathrm{~Hz}$ (Fig. 1 Bii, inset) and occurred at mean frequency of $2.5 \pm 0.2$ bursts $/ \mathrm{min}(n=32$ pups). The bursts were mainly associated with rhythmic multiple-unit discharge (Fig. $1 B$, bottom traces) with a lock of unit activity to the negative phase of spindle burst oscillations in the superficial layers (Fig. 1C). The mean cross-correlation coefficient between oscillation peak and units was $0.48 \pm 0.16(n=9$ pups). Whereas the bursts oscillations showed similar frequency over the entire depth of the cortex, a significant $(p<0.05)$ increase in the frequency of MUA could be observed from $200 \mu \mathrm{m}$ $(0.8 \pm 0.13 \mathrm{~Hz})$ to $1000 \mu \mathrm{m}(2.3 \pm 0.03 \mathrm{~Hz})$ depth ( $n=14$ pups $)$. Both the advanced maturation of neurons and connections with increasing cortical depth as the larger size and capacitance of layer 
A

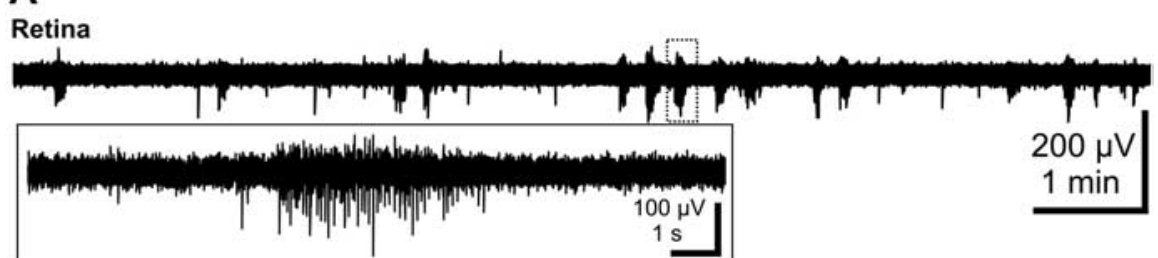

B

(i)

(ii)

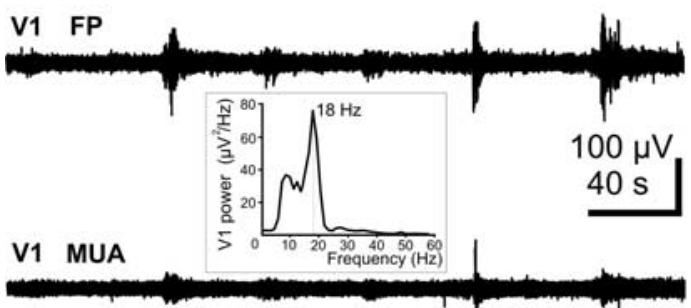

$100 \mu \mathrm{V}$

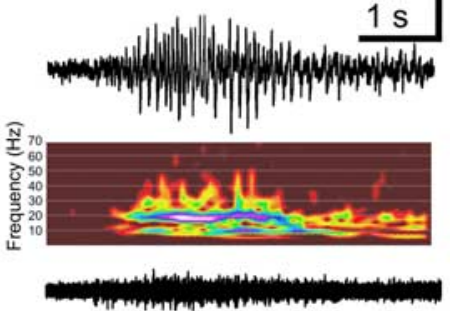

C

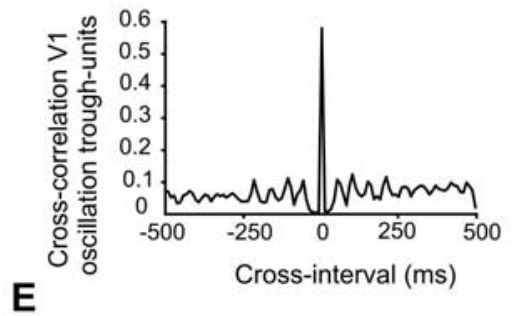

D
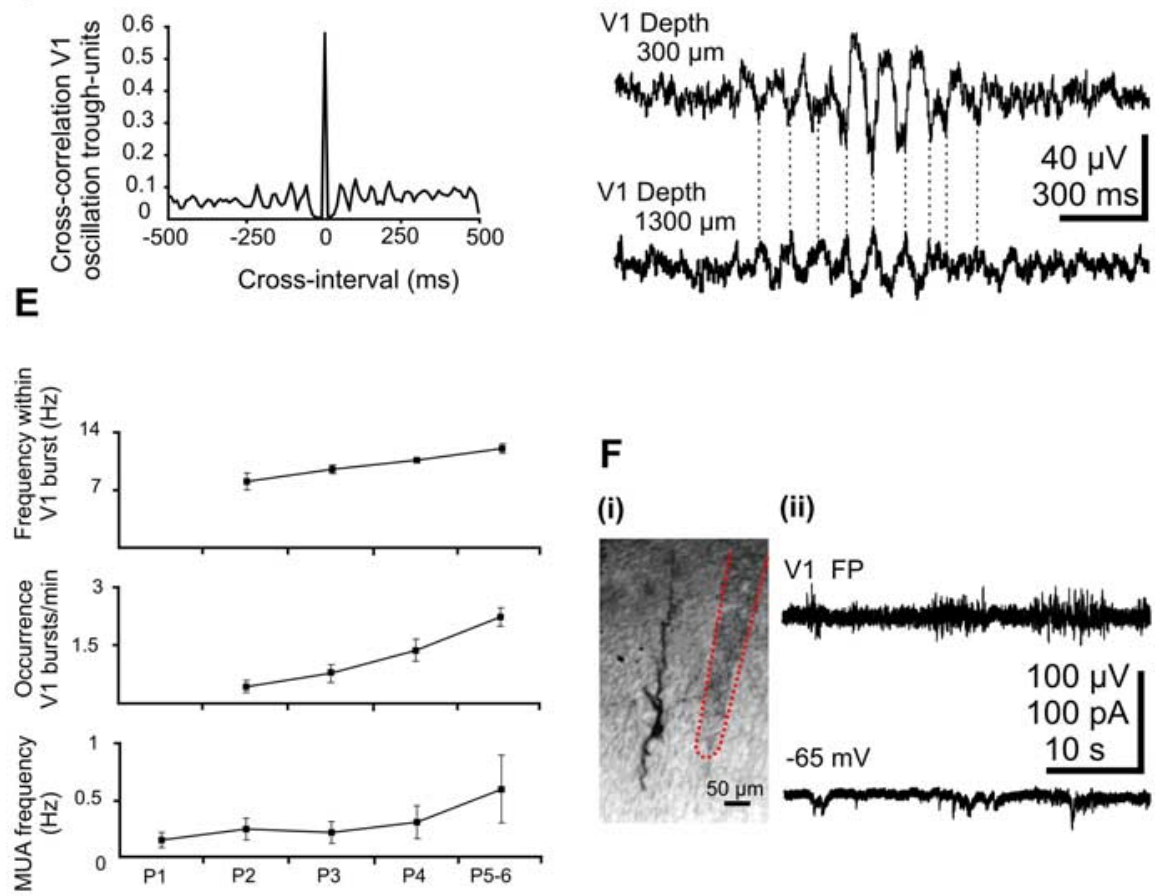

$\mathbf{F}$

(i)

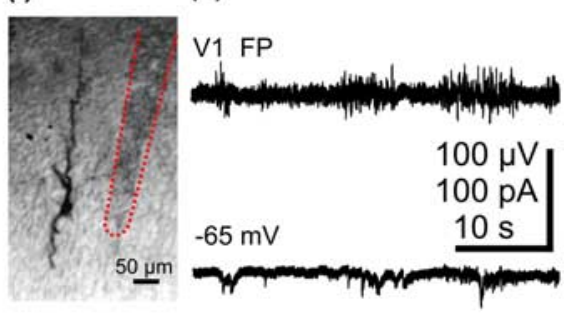

G

(i)

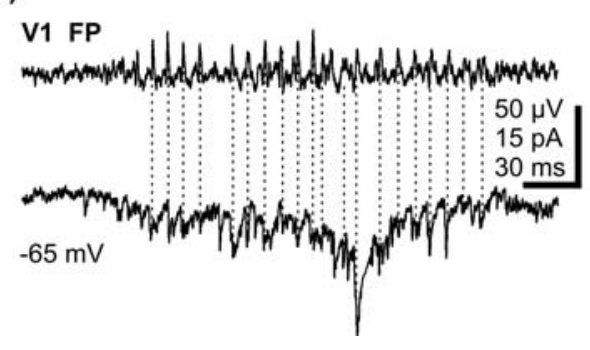

$\mathrm{V}$ and VI neurons causing action currents with larger amplitude may contribute to this increase in MUA frequency. Simultaneous recordings of the field potential in various V1 layers revealed a reversal of spindle bursts and slight decrease in their amplitude between superficial and deep layers (Fig. 1D). To assess the agedependence of oscillatory activity in the V1, we investigated the properties of bursts and MUA from P1 to P5-P6. A slight increase in the frequency of MUA from $0.14 \pm 0.07 \mathrm{~Hz}$ ( $n=4$ pups) at $\mathrm{P} 1$ to $0.6 \pm 0.3 \mathrm{~Hz}$ ( $n=4$ pups) at P5-P6 was not at a significant level. Absent at P1 $(n=$ 3), spindle bursts were observed from P2 onwards in all animals. Both the frequency of spindle bursts $(p<0.05)$ and the frequency within spindle bursts $(p<0.05)$ increased in an age-dependent manner (Fig. 1E).

To explore the synaptic mechanisms underlying the generation of network bursts in V1, patch-clamp recordings were performed in 10 P5-P6 pups. Both glutamatergic and GABAergic PSCs were recorded from predominantly pyramidalshaped cortical neurons from layers IV-V (Fig. $1 \mathrm{Fi}$ ). For the analysis of the properties of the PSCs, 30-200 individual (nonoverlapping with other events) PSCs were considered. Glutamatergic currents, recorded at the $\mathrm{GABA}_{\mathrm{A}}$ reversal potential $(-65 \mathrm{mV})$, had a mean amplitude of $13.6 \pm 1.6 \mathrm{pA}$ and occurred with a mean frequency of $0.7 \pm 0.2 \mathrm{~Hz}(n=6$ cells $)$. They were characterized by fast kinetics with a mean rise-time of $1.7 \pm 0.1 \mathrm{~ms}$ and a decay best fitted with a monoexponential function (mean $\tau, 6.2 \pm 1.7 \mathrm{~ms} ; n=6$

$\leftarrow$

D, Transcortical wide-band recordings of field activity recorded in a P6 rat. Note the phase reversal and reduction of oscillation with increasing depth. $\boldsymbol{E}$, Developmental profile of the spindle burst activity in $\mathrm{V} 1$ of newborn rat. Both the frequency of units (bottom diagram) as well as the occurrence of bursts (middle diagram) and the frequency within the spindle bursts (top diagram) significantly $(p<0.005)$ increased with age. Error bars indicate SEM. $\boldsymbol{F}$, Synaptic correlates of V1 spindle bursts. Fi, Digital stack photomontage of a whole-cell recorded and biocytin-stained pyramidal neuron from a P5 rat. The trace of the extracellular electrode close to the cell is marked by a red dotted line. Fii, Extracellular field potential recording of V1 bursts (top trace) and simultaneous voltageclamp whole-cell recording from a layer $V$ pyramidal neuron at a holding potential of $-65 \mathrm{mV}$ (bottom trace). Gi, Phaseto-phase synchronization between V1 spindle bursts and glutamatergic EPSCS voltage-clamp recorded from a layer $V$ neuron at a holding potential of $-65 \mathrm{mV}$ (the reversal potential of the $\mathrm{GABA}_{\mathrm{A}}$ receptor-mediated currents). Gii, Phase-tophase synchronization between V1 spindle burstsand GABAergic PSCs recorded at $0 \mathrm{mV}$ (the reversal potential for glutamatergic currents).
Figure 1. Burst activity in the retina and the 11 cortex of the newborn rat in vivo. $A$, Extracellular field potential recording from the retina of a $\mathrm{P} 5$ rat. Note the intermittent burst discharges separated by silent periods. Inset, Marked burst of action potentials is shown on expanded time scale. Bi, Wide-band recordings of extracellular field potential (top trace) and corresponding filtered $(0.2 \mathrm{kHz})$ MUA (bottom trace) in V1 cortex of a P5 rat. Note the correlation between field activity and MUA. Inset, Averaged power spectrum of the field potential oscillations showing maximal power at $18 \mathrm{~Hz}$. Bii, Spindle burst oscillation (top trace) and corresponding MUA (bottom trace) from the trace shown in Bi displayed at expanded time scale. Inset, Corresponding color-coded wavelet spectrum. $\boldsymbol{C}$, Normalized cross-correlograms between V1 MUA and spindle troughs from the recording shown in $\boldsymbol{B}$. 
A

(i)
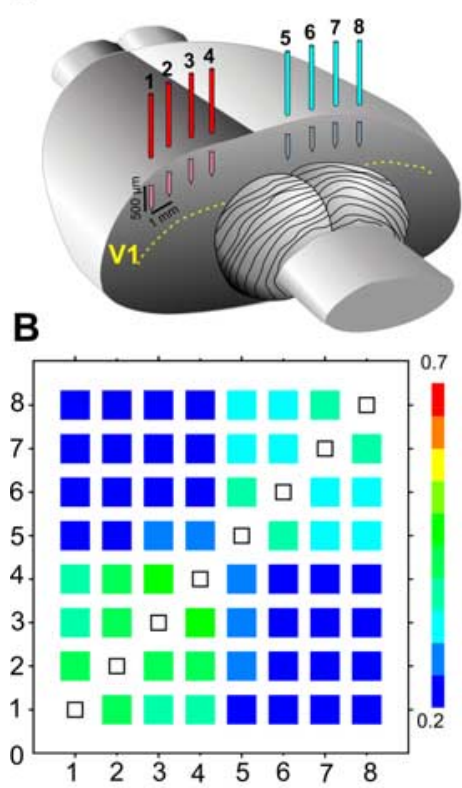

(ii)

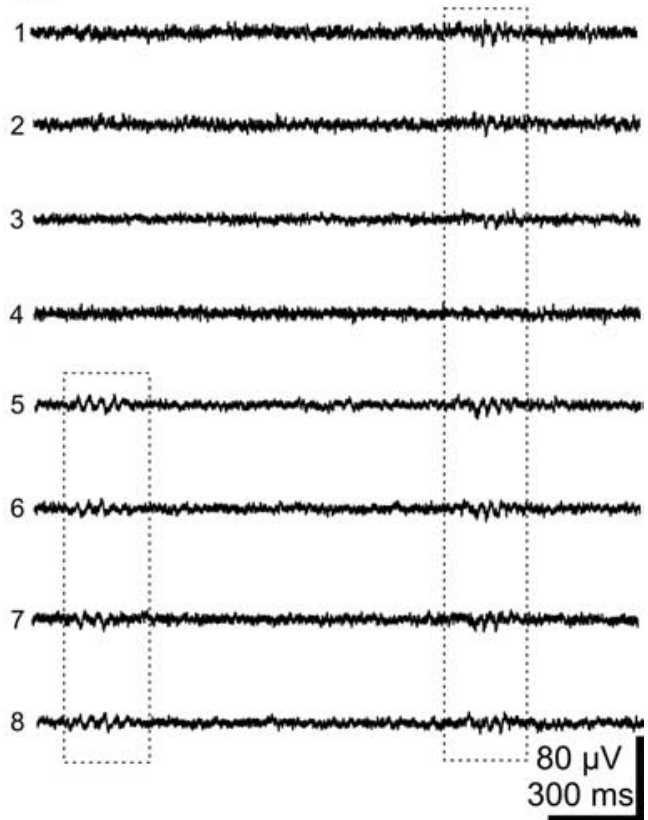

Figure 2. Spatial distribution of the spindle burst activity in V1. Ai, Illustration of the multielectrode array placed in the visual cortex. Aii, Extracellular field potential recordings performed from P6 V1 with an array of eight electrodes. Note the presence of spindle bursts synchronized over one as well as over both hemispheres. $\boldsymbol{B}$, Averaged cross-correlation matrix of bursts recorded with eight electrodes. The cross-correlation coefficients were averaged for $5-7$ bursts/electrode/pup and displayed as color-coded matrix. Note the high synchronization of bursts within one hemisphere. Data were pooled from 14 P5-P6 rats.

A
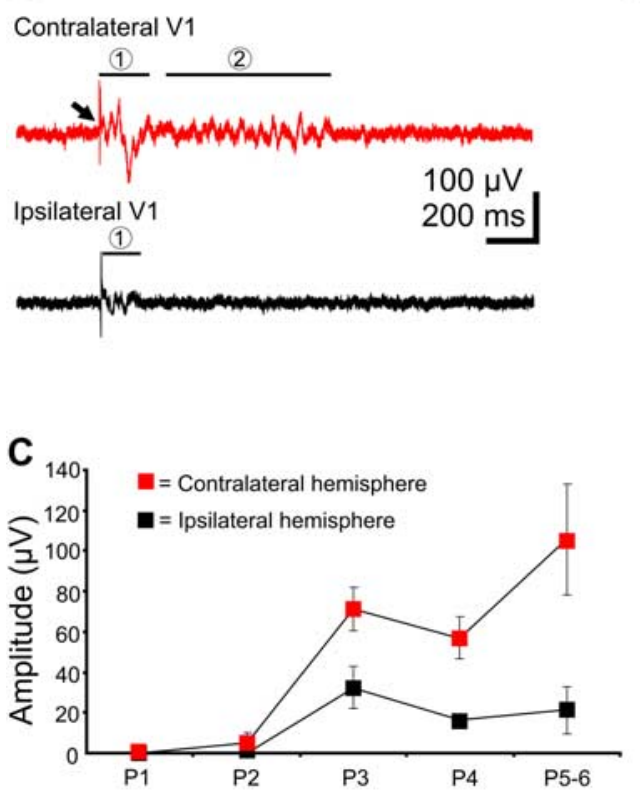

B
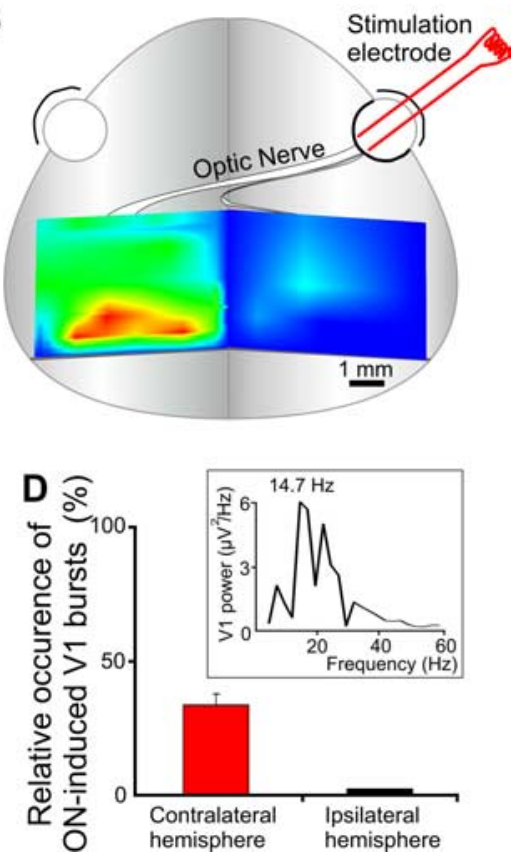

Figure 3. ON-driven activity in the V1 cortex of newborn rat. $A$, Extracellular recordings of the contralateral (top red trace) and ipsilateral (bottom black trace) cortical response to electrical stimulation of the $\mathrm{ON}$ in a $\mathrm{P} 6$ rat. Note the robust direct response (1) recorded in the contralateral hemisphere followed by spindle bursts (2), and the smaller direct response (1) on the ipsilateral side. $B$, Topographic localization of the visual cortex determined by $0 \mathrm{~N}$ responses. Multiple-site recordings were performed on both hemispheres and each direct ON-evoked response was normalized to the maximal response and color-coded (red, $100 \%$; blue, $0 \%)$. C, Developmental profile of the contralateral and ipsilateral $0 \mathrm{~N}$-evoked response. $D, 0 \mathrm{~N}$-induced oscillatory activity in the visual cortex. Bar diagram displaying the relative occurrence of $0 \mathrm{~N}$-induced spindle bursts in the ipsilateral and contralateral V1 in five investigated rats. Inset, Power spectrum of averaged ON-induced oscillations in a P5 rat. Error bars indicate SEM.

cells). The GABAergic PSCs recorded at glutamate reversal potential $(0 \mathrm{mV})$ occurred with a frequency of $0.7 \pm 0.4 \mathrm{~Hz}$ and showed a mean amplitude of $18.2 \pm$ $1.9 \mathrm{pA}(n=4$ cells $)$. They were characterized by slow kinetics and their decay was best fitted with a biexponential function $\left(\tau_{1}, 10.7 \pm 3.6 \mathrm{~ms} ; \tau_{2}, 101 \pm 42 \mathrm{~ms} ; n=4\right.$ cells). A remarkable feature of both glutamatergic and GABAergic PSCs was their grouped occurrence in bursts, which generally coincided with field spindle bursts recorded nearby by an extracellular electrode (Fig. 1 Fii,G). A relatively high degree of correlation between synaptic currents and network bursts, revealed by the cross-correlation coefficients $(0.5 \pm 0.2$ for glutamatergic PSCs versus bursts, $n=$ 6 cells; $0.5 \pm 0.1$ for GABAergic PSCs versus bursts, $n=4$ cells), supports the involvement of glutamatergic and GABAergic connections and synapses in the generation of V1 spindle bursts in P5-P6 pups.

Investigation of the spatiotemporal pattern of spindle bursts was performed using an eight-electrode array (separation distance between the electrodes $0.5-1$ $\mathrm{mm}$ ) placed on both hemispheres (Fig. $2 A i)$. In all recordings $(n=14$ P5-P6 pups), the majority of spindle bursts were either spatially confined to a single recording site or highly synchronized within one hemisphere (Fig. $2 A i i, B$ ). In contrast, only $27.1 \pm 8 \%$ of bursts showed a high degree of correlation in both hemispheres (Fig. Aii, 2B).

Cortical spindle bursts evoked by optic nerve stimulation

To first approach the role of retinal input for the generation of V1 spindle bursts, the cortical response to electrical stimulation of the ON was recorded in five pups. ON stimulation evoked a robust, mainly biphasic or triphasic response in the contralateral V1 (mean amplitude of $105.7 \pm$ $27.2 \mu \mathrm{V} ; n=5$ pups) with $26.8 \pm 1.3 \mathrm{~ms}$ delay [Fig. $3 A$, red trace, (1)]. In contrast, no detectable ( $n=1$ pup), or very small, responses (mean amplitude $21.2 \pm 11.6$ $\mu \mathrm{V} ; n=4)$ were evoked with a similar delay $(26.0 \pm 2.5 \mathrm{~ms} ; n=4$ pups $)$ in the ipsilateral V1 cortex [Fig. 3A, black trace, (1)]. ON-evoked responses were also used to determine the topography of the visual cortex in P5-P6 animals. Contralateral and ipsilateral $\mathrm{ON}$-evoked responses were recorded over a large cortical surface located between bregma and lambda and the amplitude of response was normalized to the maximal amplitude (Fig. 3B). The maximal responsive area $\left(4-5 \mathrm{~mm}^{2}\right)$ lo- 
cated along the lambda line $(0-1.5 \mathrm{~mm}$ anterior to lambda suture) corresponds to the previously established location of the visual cortex in P6-P7 pups (Olavarria and Safaeian, 2006). Therefore, all recordings included in the present study were performed in the region of maximal ON-responsiveness. In keeping with previous data (Lim and Ho, 1997), the direct response to ON stimulation was developmentally regulated. On the contralateral side, no detectable ON-response could be elicited at P1 $(n=3)$, but was observed from P2 onwards. The amplitude of the evoked responses dramatically increased from $\mathrm{P} 2$ to $\mathrm{P} 5-\mathrm{P} 6$. An ipsilateral response to $\mathrm{ON}$ stimulation was first observed at $\mathrm{P} 3$, but it was significantly $(p<0.05)$ smaller than in the contralateral cortex at all ages studied. Developmental increase in the difference between the contralateral and ipsilateral ON-responses (Fig. 3C) probably reflects gradual segregation of the optic pathways (Godement et al., 1984). An important developmental feature of the ON-evoked responses was that they were often $(33.4 \pm 4.5 \%)$ followed by spindle burst oscillatory activity in the contralateral $\mathrm{V} 1$, but not on the ipsilateral side (Fig. $3 A,(2), D)$. The ONinduced oscillations with a mean frequency of $16.9 \pm 0.6 \mathrm{~Hz}$ started $0.50 \pm 0.05 \mathrm{~s}$ after the stimulation and showed similar properties with the spontaneous V1 spindle bursts (Fig. 3D, inset). Thus, the visual pathway from the retina to the visual cortex becomes functional and its activation may trigger V1 spindle bursts during the first postnatal week.

\section{Correlation between spontaneous retinal and cortical bursts}

To decide whether retinal bursts are transmitted to the visual cortex and trigger V1 spindle bursts, we used two approaches: (1) simultaneous recordings of retinal and V1 cortical activity and (2) pharmacological modulation of retinal activity and surgical retina removal.

Simultaneous recordings from the retina and visual cortex (Fig. $4 A$ ) revealed that retinal bursts often correlate with V1 spindle bursts (Fig. $4 B, C$ ), despite the slightly lower occurrence of cortical bursts $(2.5 \pm 0.2$ bursts/min; $n=32$ pups $)$ compared with retinal bursts ( $3.1 \pm 0.7$ bursts/min; $n=6$ pups). Crosscorrelation analysis revealed robust correlation between MUA recorded from retina and MUA recorded from the contralateral V1 cortex (mean cross-correlation coefficient $0.6 \pm 0.1 ; n=5$ pups). Burst analysis revealed that a large fraction (53 $\pm 5.3 \%)$ of the contralateral cortical bursts are correlated with retinal bursts within a time window of retinal burst $\pm 1 \mathrm{~s}$ (Fig. $4 D$ ). The proportion of $\mathrm{V} 1$ spindle bursts correlated with ipsilateral retinal waves was significantly lower $(29.2 \pm 4.2 \%$; $p<0.05 ; n=3$ pups). Thus, simultaneous recordings revealed robust correlation between the retinal and contralateral V1 bursts. Because our method did not allow retinotopic recordings of retinal and cortical activity, it is possible that the level of retinocortical correlation was even underestimated (see Discussion).

Pharmacological modulation of retinal waves was achieved by intraocular injection of their activators and blockers. The acute effects on cortical activity were recorded in the contralateral and ipsilateral V1 ( $n=11$ P5-P6 pups) (Fig. $5 A, B)$. First, control experiments investigating the effect of eye operation, insertion of the application needle, and injection of solvents (saline or $0.1 \%$ DMSO) into one eye were performed. In all cases, the spindle bursts were similar in their occurrence, amplitude, and frequency to the activity recorded before eye manipulation. On the next step, we injected the agents that are known to augment retinal wave frequency and amplitude by increasing cAMP production (Tsai et al., 1987; Stellwagen et al., 1999). Slow (0.5 $\mu \mathrm{l} / \mathrm{min})$ injection of $1 \mu \mathrm{l}$ forskolin (5-10 mM) or NKH477 (water-soluble
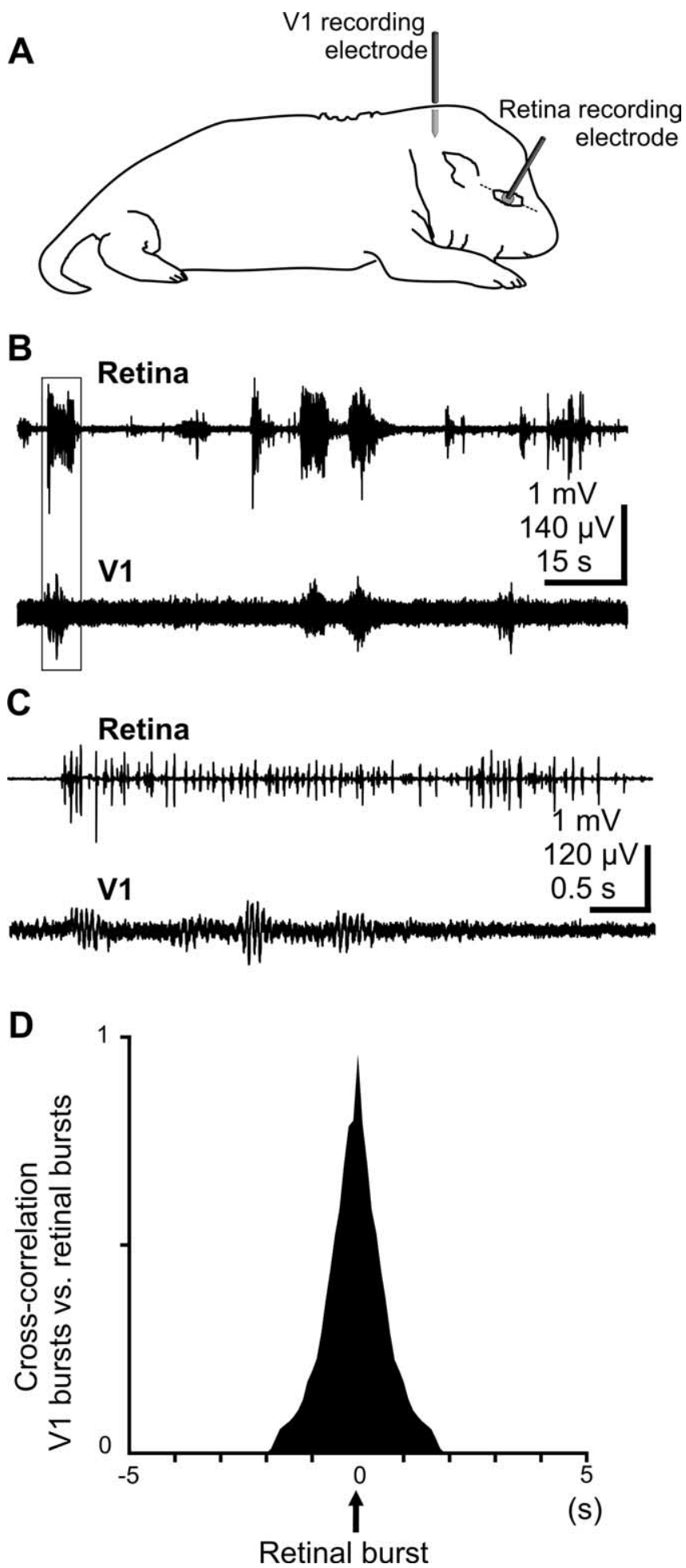

Figure 4. Correlation between retinal bursts and V1 cortical spindle bursts. $\boldsymbol{A}$, Scheme of experimental setup for simultaneous cortical and retinal recordings. $\boldsymbol{B}$, Simultaneous extracelIular local field potential recordings from retina and contralateral V1 in a P6 rat. $\boldsymbol{C}$, Strongly correlated retinal burst and $\mathrm{V} 1$ spindle bursts from the traces shown in $\boldsymbol{A}$ and displayed at an expanded time scale. $\boldsymbol{D}$, Cross-correlogram between the retinal bursts and V1 spindle bursts for four investigated pups.

forskolin, $10 \mathrm{~mm}$ ) into one eye significantly $(p<0.05)$ increased the occurrence of spindle bursts recorded in the contralateral V1. On the ipsilateral side, only a small nonsignificant increase in the burst occurrence was observed. Neither the amplitude nor the 

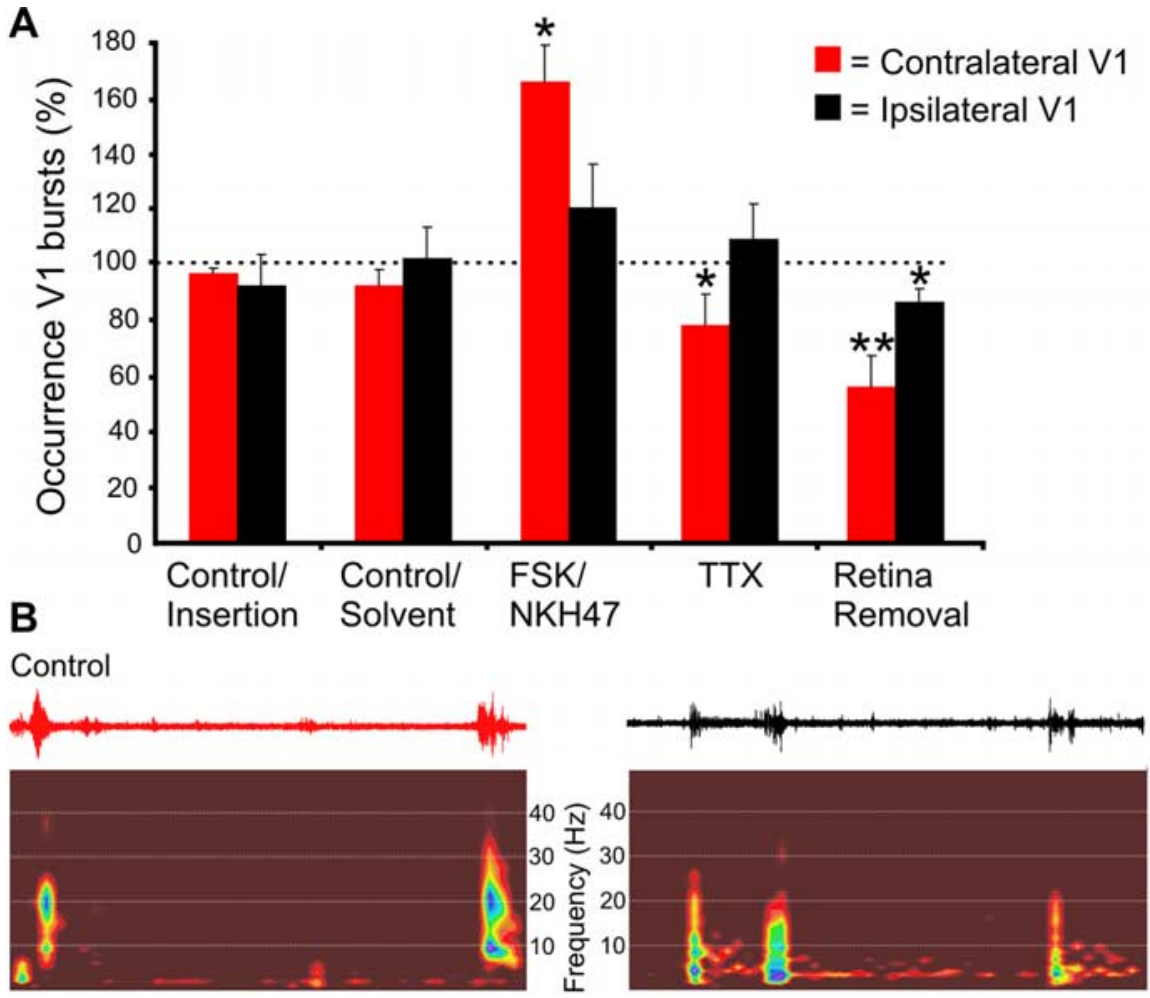

$\mathrm{NKH} 477$
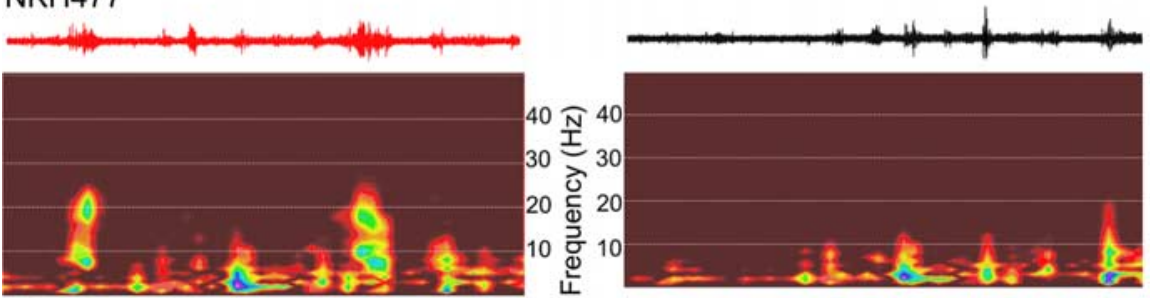

Retina removal
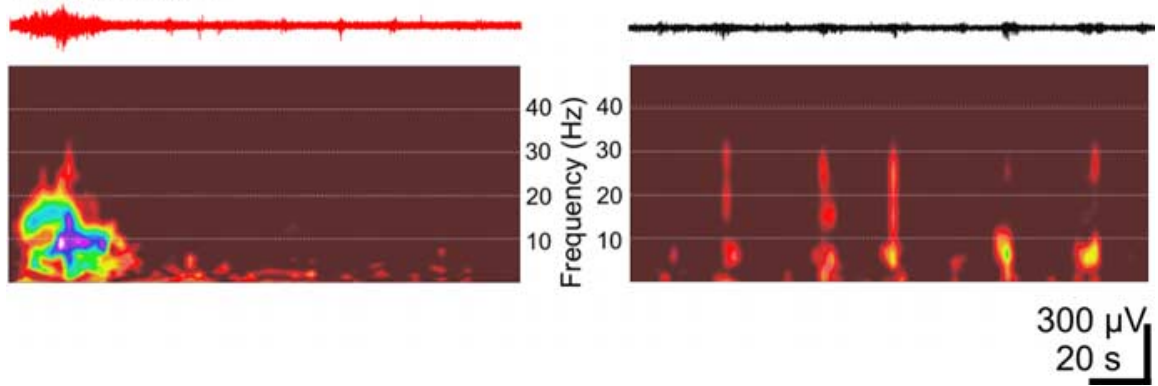

Figure 5. Effect of modification of retinal waves on V1 spindle bursts. $A$, Bar diagram of the normalized frequency of contralateral (red bars) and ipsilateral (black bars) V1 spindle bursts under intraocular insertion of the needle and solvent injection after augmentation of the retinal waves [forskolin (FSK)/NKH477], after blockade of activity propagation (TTX), and after retina removal ( $n=11$ pups). Error bars indicate SEM. B, Extracellular recordings of the contralateral (red traces) and ipsilateral (black traces) V1 spindle bursts in a P5 rat and the corresponding color-coded wavelet spectra under control conditions after injection of NKH477 and after retina removal.

tralateral bursts without any effect on the ipsilateral activity ( $n=7$ pups). Similar negative results were obtained when injecting a mixture of $200 \mu \mathrm{M} \mathrm{CPP}$ and 100 $\mu \mathrm{M}$ CNQX to block the glutamatergic receptors ( $n=3$ pups). These negative results could be attributable to uncertainty of the efficient dose of these drugs in vivo. Therefore, we used a more radical approach to abolish the retinal output by blocking action potential transmission via the optic nerve. Because previous in vivo data on cats reported that an abolishment of the retinal waves with TTX was not possible without being lethal for the animals (Greuel et al., 1987; Stellwagen and Shatz, 2002), the injection needle was slightly advanced to pierce the retina and to directly reach the head of the ON. Blockade of action potential propagation with TTX (10$200 \mu \mathrm{M})$ led to a significant $(p<0.05)$ decrease in the frequency of contralateral bursts but had no effect on the ipsilateral side ( $n=8$ pups). At the end of experiment, the retina was removed under local anesthesia ( $n=6$ pups). After a $30-60$ min recovery period, a nearly twofold $(p<0.01)$ decrease in the occurrence frequency of the contralateral bursts could be observed, together with a slight decrease $(p<0.05)$ in the ipsilateral oscillatory activity. Removal of both retinas $(n=3$ pups) had no additional effect on the cortical activity of one hemisphere (55.4 \pm $11.5 \%$ burst activity left after removal of one retina; $51 \pm 5 \%$ burst activity left after removal of both retinas), whereas the occurrence frequency of bursts was significantly $(p<0.01)$ decreased on the opposite hemisphere $(85.8 \pm 4.8 \%$ burst activity left after removal of one retina and $46.4 \pm 3.6 \%$ burst activity left after removal of both retinas). These results indicate that retinal activity modulates the cortical pattern of activity, but it is not a necessary condition for generation of $\mathrm{V} 1$ spindle bursts.

\section{Discussion}

In the present study, we examined the hypothesis that retinal waves are transmitted to the visual cortex and drive cortical activity patterns. Using recordings from the retina and visual cortex of neonatal rats in

frequency of cortical bursts was significantly modified by the cAMP activators. Depending on the postnatal developmental stage, retinal waves in vitro can be abolished by blocking either nicotinic acetylcholine receptors (nAChRs) (Feller et al., 1996) or ionotropic glutamate receptors (Zhou and Zhao, 2000). We therefore attempted to use acetylcholine and glutamate receptor antagonists in vivo. However, injection of the $\mathrm{nAChR}$ antagonist dehydro- $\beta$-erythroidine at high concentration $(200 \mu \mathrm{M})$ only slightly and nonsignificantly decreased the occurrence of con- vivo, we have shown that (1) the dominant pattern of activity in the immature visual cortex is a spontaneous spindle burst defined as an intermittent, spatially confined network burst of multiple unit activity associated with spindle-shape field oscillations; (2) spindle bursts in visual cortex are correlated with spontaneous retinal bursts, and can be evoked by stimulation of the optic nerve; (3) pharmacological modulation of retinal activity affects the frequency of V1 spindle bursts; and (4) blockade of propaga- 
tion of retinal activity or removal of the retina reduces but does not eliminate cortical spindle bursts. Thus, these results suggest that retinal activity is transmitted via the LGN to the visual cortex and triggers endogenous V1 spindle bursts.

\section{Retinal control of V1 spindle bursts}

Several lines of evidence indicate that retinal waves propagate to the visual cortex and drive V1 spindle burst oscillations. First, we found that direct optic nerve stimulation evokes a response in the visual cortex. This indicates that the entire visual pathway from the retina, through the LGN, to the visual cortex is already functional during the first postnatal week. Sensory response to optic nerve stimulation was often followed by spindle bursts. Second, we found robust correlation between retinal and cortical activities. Not all retinal bursts were correlated with cortical spindle bursts and retinal activity did not significantly precede the cortical events that could have been expected if the retina drove cortical bursts. This is likely because of the limitations of the technique used in this study, which does not enable retinotopic recordings from retina and visual cortex because the cortical electrode was likely located beside the retinotopic projection of the retinal electrode. In our opinion, the correlation found between retinal and cortical bursts as well as MUA rather reflects a retinal wave, which relatively slowly spreads across the retina, and often through only part of the retina, and activates the cortex corresponding to the retinotopic projection and retinal spread. Thus, retinal drive to various areas of the visual cortex a priori should not be synchronous and it should respect the spread of retinal activity. Additional studies using multielectrode arrays or imaging from the retina and visual cortex in vivo are required to address the precise retinotopic dynamics of cortical activity. Third, evidence supporting the control of cortical activity by retinal waves is the modulation of the frequency of spindle bursts by pharmacological manipulations that are known to modify the occurrence of retinal waves. Intraocular forskolin injection, which increases the frequency and amplitude of retinal waves (Tsai et al., 1987), strongly increased the occurrence of the cortical spindle bursts in the contralateral V1 cortex. In addition, removal of the retina resulted in a twofold reduction of V1 spindle bursts frequency. These results indicate that retinal activity is transmitted to the cortex and drives an endogenous pattern of V1 spindle bursts. However, retinal drive is not a necessary condition for V1 spindle burst generation because they persisted, although at lower frequency, after deafferentation.

Similar to the activity reported here, spindle bursts are also present at later stages of development, when the retina becomes responsive to light. In P22-P39 ferrets, V1 multiple-unit activity is organized in bursts with $\sim 10 \mathrm{~Hz}$ intraburst multiple-unit activity and these bursts can be evoked by light stimulation. Studied with multielectrode arrays, this bursting activity shows a patchy structure that reflects ocular dominance columns in the visual cortex (Chiu and Weliky, 2001; Chiu and Weliky, 2002). In keeping with these results in ferrets, we also found that later during rat development (by P10), spindle bursts can be evoked in the visual cortex by light stimulation (R. Khazipov, unpublished observations). Generally, the later visual experience is able to affect the architecture of intracortical circuits and to shape the development of visual cortex (White et al., 2001).

\section{Physiological roles for retina driven V1 cortical bursts}

Very recently, evidence was provided that patterned retinal activity during the first week of life is essential for organizing precise connections to the visual cortex (Cang et al., 2005). In adult mice in which spontaneous activity in the retina during the first postnatal week was disrupted genetically or pharmacologically, opti- cal imaging and microelectrode recordings revealed imprecise anatomical mapping of the geniculocortical projections. The deficits were already present by P8 and removing the eyes afterward had no effect on the projection patterns. Our finding of the retinal control of V1 spindle bursts during the first postnatal week may provide a physiological basis for this plasticity. We propose that the retinal wave-driven and spontaneous V1 spindle bursts contribute to the activity-dependent formation and refinement of precise thalamocortical connections. At the cellular level, activation of the retinal wave-driven thalamocortical input may be boosted by the following spindle burst depolarization and thereby provides conditions for Hebbian plasticity at these developing synapses (Crair and Malenka, 1995; Feldman et al., 1998, 1999). A similar paradigm has been proposed during a comparable stage of somatosensory cortex development (Khazipov et al., 2004).

\section{Human correlates}

Cortical maturity in the rat during the first postnatal week corresponds to the young premature human (Gottlieb et al., 1977; Dobbing and Sands, 1979; Clancy et al., 2001; Khazipov et al., 2001). Temporal organization of cortical electroencephalogram at occipital recordings from the visual cortex in early prematurity is characterized by bursts of activity typically organized in spindle-like oscillations (5-25 Hz) superimposed on delta waves (delta brushes) (Dreyfus-Brisac and Larroche, 1971; StockardPope et al., 1992; Lamblin et al., 1999). V1 spindle bursts in neonatal rats are highly reminiscent of these premature human occipital delta brushes. Interestingly, human occipital delta brushes appear later in development than delta brushes in the central cortex, and rat V1 spindle bursts emerge later (P2) than spindle bursts in the somatosensory cortex (P1) (I. L. Hanganu, unpublished observations). This delay between S1 and V1 probably reflects the rostrocaudal gradient of cortical development. Spontaneous retinal activity also participates in cortical development in primates. In the retina-ablated macaque embryos, some basic aspects of the cytoarchitectonic and chemoarchitectonic organization of the cerebral cortex and certain parameters of synaptic development develop to an optimal normal level (Kuljis and Rakic, 1990; Bourgeois and Rakic, 1996). However, in the thalamorecipient sublayers, the localization on dendritic spines or shafts fails to mature properly in the absence of normal functional input from the periphery (Bourgeois and Rakic, 1996). This raises an intriguing hypothesis that delta brushes can be triggered by spontaneous retinal waves, which provide the input to the visual cortex in utero and drive developmental plasticity in the absence of visual sensory input.

\section{Spindle burst is the principal activity pattern in the immature cortex}

In the present study, we demonstrate for the first time that the spindle burst is the principal pattern of coordinated activity expressed in the neonatal rat visual cortex in vivo. The pattern of spindle bursts has been initially described in the somatosensory cortex of the rat during the first postnatal week (Khazipov et al., 2004). There are a number of similarities between the spindle bursts in the somatosensory and visual cortices, including the dominant frequency of the intraburst oscillations at $\sim 10-15 \mathrm{~Hz}$, spindle shape, duration of $\sim 1 \mathrm{~s}$, depth profile with the reversal at around the cortical layer IV, time lock of neuronal firing to the negative phase of the oscillations in the superficial layers, and participation of glutamatergic and GABAergic synapses. Both S1 and V1 spindle bursts were reliably triggered by sensory stimulation and were reduced in frequency, but not totally blocked, by 
deafferentation. In both systems, input from the periphery is transmitted via the thalamus to the cortex and triggers an endogenous cortical pattern of spindle bursts. Thus, early in development, both systems operate in a similar mode in which spontaneous activity at the periphery drives a cortical oscillator. It remains to be determined whether this principle of function is general for all developing sensory systems.

Although mechanisms underlying spindle bursts are not completely understood, their generation likely involves interaction between (1) endogenous (including cortical and thalamocortical), (2) periphery-driven, and (3) neuromodulatory (e.g., cholinergic) systems. Studies in the somatosensory system revealed rhythmic activity of neurons in the ventroposterolateral thalamus at spindle-burst frequency, as well as a correlation between the thalamic and $\mathrm{S} 1$ cortical activity and movements (Khazipov et al., 2004). Thus, the thalamus is clearly involved as a relay station to convey the activity from the periphery to the cortex and to trigger spindle bursts. However, except for this function, the thalamus seems to play a rather limited role in the generation of cortical oscillations, as suggested by several lines of evidence: (1) pharmacological blockade or cut of the optic nerve suppresses LGN bursts in the intact retina/LGN preparation in vitro (Mooney et al., 1996), (2) optic nerve cut transiently suppresses LGN but not cortical bursts in vivo (Weliky and Katz, 1999; Chiu and Weliky, 2001), and (3) in the mouse thalamocortical slices preparation, spindle-like oscillations cannot be evoked by internal capsule stimulation before P12 (Warren et al., 1994; Warren and Jones, 1997). However, in vitro evidence exists that isolated neonatal cortex is capable of generating spindle-shape oscillations at $\beta$-gamma frequency very similar to the spindle bursts in vivo (Kilb and Luhmann, 2003; Dupont et al., 2006). An interesting property of these in vitro spindle-shape oscillations is that they are acetylcholine-dependent and completely blocked by the muscarinic acetylcholine receptor antagonists. Whereas directly after birth (P1-P3) the spindle-shape oscillations are synchronized via gap junctions, synaptic transmission starts to play the main role in their generation around P5-P6, as demonstrated by the in vivo data in S1 (Khazipov et al., 2004) and V1 (present study) and by the in vitro results (Dupont et al., 2006). Taking into account these in vitro and in vivo observations, we propose that spindle-burst is an endogenous cortical pattern, which is controlled by subcortical cholinergic input and can also be triggered by the input from the periphery. Elucidation of spindle burst mechanisms in sensory systems and decisions on the roles of the gap-junctions, synaptic connections, and cholinergic input in the generation and propagation of oscillatory activity in vivo represent challenging tasks for the future.

\section{References}

Ben-Ari Y (2001) Developing networks play similar melody. Trends Neurosci 24:354-360.

Bourgeois JP, Rakic P (1996) Synaptogenesis in the occipital cortex of Macaque monkey devoid of retinal input from early embryonic stages. Eur J Neurosci 8:942-950.

Cang J, Renteria RC, Kaneko M, Liu X, Copenhagen DR, Stryker MP (2005) Development of precise maps in visual cortex requires patterned spontaneous activity in the retina. Neuron 48:797-809.

Chandrasekaran AR, Plas DT, Gonzalez E, Crair MC (2005) Evidence for an instructive role of retinal activity in retinotopic map refinement in the superior colliculus of the mouse. J Neurosci 25:6929-6938.

Chiu C, Weliky M (2001) Spontaneous activity in developing ferret visual cortex in vivo. J Neurosci 21:8906-8914.

Chiu C, Weliky M (2002) Relationship of correlated spontaneous activity to functional ocular dominance columns in the developing visual cortex. Neuron 35:1123-1134.
Clancy B, Darlington RB, Finlay BL (2001) Translating developmental time across mammalian species. Neuroscience 105:7-17.

Crair MC, Malenka RC (1995) A critical period for long-term potentiation at thalamocortical synapses. Nature 375:325-328.

Crowley JC, Katz LC (1999) Development of ocular dominance columns in the absence of retinal input. Nat Neurosci 2:1125-1130.

Dobbing J, Sands J (1979) Comparative aspects of the brain growth spurt. Early Hum Dev 3:79-83.

Dreyfus-Brisac C, Larroche JC (1971) Discontinuous electroencephalograms in the premature newborn and at term. Electro-anatomo-clinical correlations (in French). Rev Electroencephalogr Neurophysiol Clin 1:95-99.

Dupont E, Hanganu IL, Kilb W, Hirsch S, Luhmann HJ (2006) Rapid developmental switch in the mechanisms driving early cortical columnar networks. Nature 439:79-83.

Feldman DE, Nicoll RA, Malenka RC, Isaac JT (1998) Long-term depression at thalamocortical synapses in developing rat somatosensory cortex. Neuron 21:347-357.

Feldman DE, Nicoll RA, Malenka RC (1999) Synaptic plasticity at thalamocortical synapses in developing rat somatosensory cortex: LTP, LTD, and silent synapses. J Neurobiol 41:92-101.

Feller MB, Wellis DP, Stellwagen D, Werblin FS, Shatz CJ (1996) Requirement for cholinergic synaptic transmission in the propagation of spontaneous retinal waves. Science 272:1182-1187.

Firth SI, Wang CT, Feller MB (2005) Retinal waves: mechanisms and function in visual system development. Cell Calcium 37:425-432.

Galli L, Maffei L (1988) Spontaneous impulse activity of rat retinal ganglion cells in prenatal life. Science 242:90-91.

Godement P, Salaun J, Imbert M (1984) Prenatal and postnatal development of retinogeniculate and retinocollicular projections in the mouse. J Comp Neurol 230:552-575.

Gottlieb A, Keydar I, Epstein HT (1977) Rodent brain growth stages: an analytical review. Biol Neonate 32:166-176.

Greuel JM, Luhmann HJ, Singer W (1987) Evidence for a threshold in experience-dependent changes of kitten visual cortex. Dev Brain Res 34:141-149.

Grubb MS, Rossi FM, Changeux JP, Thompson ID (2003) Abnormal functional organization in the dorsal lateral geniculate nucleus of mice lacking the beta 2 subunit of the nicotinic acetylcholine receptor. Neuron 40:1161-1172.

Katz LC, Shatz CJ (1996) Synaptic activity and the construction of cortical circuits. Science 274:1133-1138.

Khazipov R, Esclapez M, Caillard O, Bernard C, Khalilov I, Tyzio R, Hirsch J, Dzhala V, Berger B, Ben-Ari Y (2001) Early development of neuronal activity in the primate hippocampus in utero. J Neurosci 21:9770-9781.

Khazipov R, Sirota A, Leinekugel X, Holmes GL, Ben Ari Y, Buzsaki G (2004) Early motor activity drives spindle bursts in the developing somatosensory cortex. Nature 432:758-761.

Kilb W, Luhmann HJ (2003) Carbachol-induced network oscillations in the intact cerebral cortex of the newborn rat. Cereb Cortex 13:409-421.

Kuljis RO, Rakic P (1990) Hypercolumns in primate visual cortex can develop in the absence of cues from photoreceptors. Proc Natl Acad Sci USA 87:5303-5306.

Lamblin MD, Andre M, Challamel MJ, Curzi-Dascalova L, d'Allest AM, De Giovanni E, Moussalli-Salefranque F, Navelet Y, Plouin P, RadvanyiBouvet MF, Samson-Dollfus D, Vecchierini-Blineau MF (1999) Electroencephalography of the premature and term newborn. Maturational aspects and glossary. Neurophysiol Clin 29:123-219.

Leinekugel X, Khazipov R, Cannon R, Hirase H, Ben Ari Y, Buzsaki G (2002) Correlated bursts of activity in the neonatal hippocampus in vivo. Science 296:2049-2052.

Lim CH, Ho SM (1997) Early detection of optic nerve-evoked response in the superior colliculus of the neonatal rat. Neurosci Lett 235:141-144.

Margrie TW, Brecht M, Sakmann B (2002) In vivo, low-resistance, wholecell recordings from neurons in the anaesthetized and awake mammalian brain. Pflugers Arch 444:491-498.

McLaughlin T, Torborg CL, Feller MB, O'Leary DD (2003) Retinotopic map refinement requires spontaneous retinal waves during a brief critical period of development. Neuron 40:1147-1160.

Meister M, Wong RO, Baylor DA, Shatz CJ (1991) Synchronous bursts of action potentials in ganglion cells of the developing mammalian retina. Science 252:939-943. 
Moody WJ, Bosma MM (2005) Ion channel development, spontaneous activity, and activity-dependent development in nerve and muscle cells. Physiol Rev 85:883-941.

Mooney R, Penn AA, Gallego R, Shatz CJ (1996) Thalamic relay of spontaneous retinal activity prior to vision. Neuron 17:863-874.

Mrsic-Flogel TD, Hofer SB, Creutzfeldt C, Cloez-Tayarani I, Changeux JP, Bonhoeffer T, Hubener M (2005) Altered map of visual space in the superior colliculus of mice lacking early retinal waves. J Neurosci 25:6921-6928.

Muir-Robinson G, Hwang BJ, Feller MB (2002) Retinogeniculate axons undergo eye-specific segregation in the absence of eye-specific layers. J Neurosci 22:5259-5264.

Olavarria JF, Safaeian P Development of callosal topography in visual cortex of normal and enucleated rats. J Comp Neurol, in press.

Penn AA, Riquelme PA, Feller MB, Shatz CJ (1998) Competition in retinogeniculate patterning driven by spontaneous activity. Science 279:2108-2112.

Rakic P (1976) Prenatal genesis of connections subserving ocular dominance in the rhesus monkey. Nature 261:467-471.

Shatz CJ, Stryker MP (1988) Prenatal tetrodotoxin infusion blocks segregation of retinogeniculate afferents. Science 242:87-89.

Stellwagen D, Shatz CJ (2002) An instructive role for retinal waves in the development of retinogeniculate connectivity. Neuron 33:357-367.

Stellwagen D, Shatz CJ, Feller MB (1999) Dynamics of retinal waves are controlled by cyclic AMP. Neuron 24:673-685.

Stockard-Pope JE, Werner SS, Bickford RG (1992) Atlas of neonatal electroencephalography, Ed 2. New York: Raven.
Stryker MP, Harris WA (1986) Binocular impulse blockade prevents the formation of ocular dominance columns in cat visual cortex. J Neurosci 6:2117-2133.

Torborg CL, Feller MB (2005) Spontaneous patterned retinal activity and the refinement of retinal projections. Prog Neurobiol 76:213-235.

Tsai SC, Noda M, Adamik R, Moss J, Vaughan M (1987) Enhancement of choleragen ADP-ribosyltransferase activities by guanyl nucleotides and a 19 kDa membrane protein. Proc Natl Acad Sci USA 84:5139-5142.

Warren RA, Jones EG (1997) Maturation of neuronal form and function in a mouse thalamocortical circuit. J Neurosci 17:277-295.

Warren RA, Agmon A, Jones EG (1994) Oscillatory synaptic interactions between ventroposterior and reticular neurons in mouse thalamus in vitro. J Neurophysiol 72:1993-2003.

Weliky M, Katz LC (1999) Correlational structure of spontaneous neuronal activity in the developing lateral geniculate nucleus in vivo. Science 285:599-604.

White LE, Coppola DM, Fitzpatrick D (2001) The contribution of sensory experience to the maturation of orientation selectivity in ferret visual cortex. Nature 411:1049-1052.

Wong RO, Meister M, Shatz CJ (1993) Transient period of correlated bursting activity during development of the mammalian retina. Neuron 11:923-938.

Wong RO, Chernjavsy A, Smith SJ, Shatz CJ (1995) Early functional neural networks in the developing retina. Nature 374:716-718.

Zhou ZJ, Zhao D (2000) Coordinated transitions in neurotransmitter systems for the initiation and propagation of spontaneous retinal waves. J Neurosci 20:6570-6577. 